\title{
Spinal Deformity Surgery: A Critical Review of Alignment and Balance
}

\author{
Matthias Pumberger ${ }^{1,2}$, Hendrik Schmidt ${ }^{3}$, Michael Putzier ${ }^{1}$ \\ ${ }^{1}$ Spine Department, Center for Musculoskeletal Surgery, Charité - Universitätsmedizin Berlin, Berlin, Germany \\ ${ }^{2}$ Berlin-Brandenburg School for Regenerative Therapies, Charité - Universitätsmedizin Berlin, Berlin, Germany \\ ${ }^{3}$ Julius Wolff Institute, Charité - Universitätsmedizin Berlin, Berlin, Germany
}

Correction of the overall coronal and/or sagittal plane deformities is one of the main predictors of successful spinal surgery. In routine clinical practice, spinal alignment is assessed using several spinal and pelvic parameters, such as pelvic incidence and tilt, sacral slope, lumbar lordosis, thoracic kyphosis, and sagittal vertical axis. Standard values have been defined for all these parameters, and the formulas of correction have been set for determining the surgical strategy. However, several factors can potentially bias these formulas. First, all standard values are measured using conventional plain radiographs and are, therefore, prone to bias. The radiologist, measuring surgeon, and patient are possible confounding influencing factors. Second, spino-pelvic compensatory effects and biomechanically relevant structures for the patient's posture, including ligaments, tendons, and muscles, have received minimal consideration in the literature. Therefore, even in cases of appropriately planned deformity correction surgeries, complications, revision rates, and surgical outcomes significantly vary. This study aimed to illustrate the current clinical weaknesses of the assessment of spinal alignment and the importance of holistically approaching the musculoskeletal system for any spinal deformity surgery. We believe that our detailed insights regarding spinal, sagittal, and coronal alignments as well as the considerations of an individual's spinal balance will contribute toward improvement in routine patient care.

Keywords: Sagittal balance; Adult spinal deformity; Spinal surgery; Spine biomechanics

\section{Introduction}

In the classic anatomical literature, the shape of the lumbar spine was considered to be standardized and uniformly lordotic between the first and fifth lumbar vertebrae [1]. However, recent radiological studies have revolutionized the anatomical and clinical understanding of the lumbar spine and have demonstrated that whole spino-sacral morphology and orientation may considerably vary even in asymptomatic patients [2,3]. Early studies have demon- strated that, in the standing position, the spatial orientation and morphology of different anatomical entities (e.g., the femur, pelvic bones, sacrum, and lumbar spine) are closely interrelated and those of one entity affect those of the adjacent entities $[3,4]$. For example, the individual pelvic incidence is closely correlated with the sacral slope and pelvic tilt and, thus, with the individual amount of lumbar lordosis.

The interrelationships among the femur, pelvic bones, sacrum, and lumbar spine $[3,4]$ result in different sugges-

Received Jun 29, 2017; Revised Oct 23, 2017; Accepted Nov 12, 2017

Corresponding author: Matthias Pumberger

Spine Department, Center for Musculoskeletal Surgery, Charitéplatz 1, 10117 Berlin, Germany

Tel: +49-30-450-652156, Fax: +49-30-450-552079, E-mail: matthias.pumberger@charite.de 
tions for the classifications of sagittal alignment. Roussouly et al. [5] proposed one of the most influential and widely employed classification systems based on four elementary types of sagittal alignment that differ, for example, in the sacral slope and the resultant segmental and total amount of lordosis [5]. A recent study has shown that these anatomical interrelationships and the resultant classification systems, commonly called "sagittal balance" [6], have major implications for the pathogenesis of different degenerative pathologies and their treatment. Therefore, standard values for all these parameters have been defined, and formulas of correction have been established to help make a decision regarding the surgical strategy. However, several factors can potentially bias these formulas and values.

The purpose of this study was to illustrate the current strategies for the assessment of spinal deformities in clinical practice and patient outcomes after corrective surgery. Further, we identified relevant misconceptions and limitations that may explain the unsatisfactory clinical results. Further, to overcome the existing limitations, functional analyses that should be considered while deciding the surgical strategy are discussed.

\section{Current Strategy for Determining Sagittal Spinal Alignment and Surgical Correction}

Physiological spinal alignment, first introduced by Vaz et al. [3], is defined as a balanced position between the pelvis and the spine in the sagittal and coronal planes, as that while standing with the knees and hips comfortably extended, the shoulders neutral or flexed, the neck neutral, and the gaze horizontal. To standardize the capture of the current state of a patient's alignment or misalignment $[7,8]$ and more precisely assign patients to appropriate treatments $[9,10]$, recent studies have defined different anatomical parameters such as pelvic incidence, pelvic tilt $[11,12]$, vertical plumb line [13-15], lumbar lordosis [16$18]$, and sacrum orientation $[19,20]$. Pelvic incidence is a measure of the sagittal orientation of the sacrum relative to the acetabula and is independent of posture (Fig. 1). It is stable in adults; however, inter-individual differences exist. In contrast, all other parameters can compensate, depending on the spinal deformity. These parameters have been previously investigated, particularly during the last decade, and several authors have defined standard values defining the optimal balance for spinal alignment. The fol-

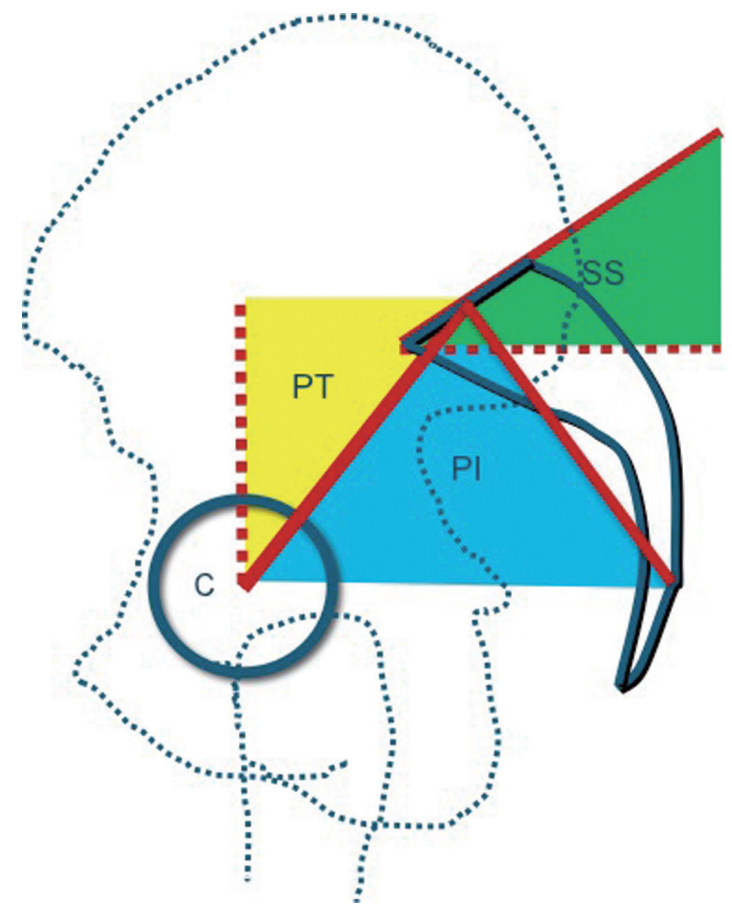

Fig. 1. The figure illustrates the most relevant spino-pelvic clinical parameters measured using conventional lateral radiography. PT (yellow) is the angle of the vertical line from the hip joint center and the line to the sacral plateau; SS (green) is the angle of the horizontal line and sacral plateau; and PI (blue) is the angle between the perpendicular of the midpoint of the sacral plateau and the line to the sacral plateau. PT, pelvic tilt; SS, sacral slope; PI, pelvic incidence.

lowing spino-pelvic parameters are suggested for healthy adults without any spinal disorder, describing a spinal sagittal balance [21,22]: pelvic incidence, $56^{\circ} \pm 10^{\circ}$ for women and $53^{\circ} \pm 10.6^{\circ}$ for men; sacral slope, $43.2^{\circ} \pm 8.4^{\circ}$ for women and $41^{\circ} \pm 8.5^{\circ}$ for men; pelvic tilt, $13.6^{\circ} \pm 6^{\circ}$ for women and $13^{\circ} \pm 6^{\circ}$ for men; and lumbar lordosis, $36.1^{\circ} \pm 13.2^{\circ}$ for women and $30.5^{\circ} \pm 8.2^{\circ}$ for men. Similar findings were reported by Vaz et al. [3] who found a statistically significant correlation (Pearson's bilateral test) between pelvic incidence and sacral slope $(r=0.86)$, pelvic incidence and lumbar lordosis $(r=0.69)$, pelvic incidence and pelvic tilt $(r=0.59)$, sacral slope and lumbar lordosis $(\mathrm{r}=0.75)$, and lumbar lordosis and thoracic kyphosis $(r=0.36)$.

To evaluate the global spinal balance, different measurements have been proposed. The most commonly used index is the C7 plumb line, which is a vertical line originating at the center of the $\mathrm{C} 7$ vertebral body with respect to the posterior superior corner of S1 [13]. This line should pass through the superior endplate of $\mathrm{S1}$, or more precisely within approximately $2 \mathrm{~cm}$ of the posterosuperior corner of the S1 vertebral body $[23,24]$. The position 
of this line is then termed positive (the plumb line passes $>2 \mathrm{~cm}$ in front of the posterosuperior corner of the S1), neutral (the plumb line passes within $2 \mathrm{~cm}$ in front of the posterosuperior corner of the $\mathrm{S} 1$ ) or negative (the plumb line passes $>2 \mathrm{~cm}$ behind the posterosuperior corner of the S1).

Current investigations on patients with spinal deformities suggest a close relationship between the aforementioned balance parameters and the quality of life (QoL) of patients [25]. Knowledge on the QoL aspects that are most affected by a particular disease helps researchers and clinicians in identifying disease-related problems that may be inadvertently omitted in research and clinical practice. Recently, methods for calculating the extent of deformity correction have been published (e.g., the exact method of Ondra, the FBI method of Le Huec, and the spinofemoral angle method of Lamartina), suggesting invasive procedures such as osteotomies to achieve the complete restoration of spinal alignment [26]. These methods have been demonstrated to yield different preoperative values of lordosis correction [26]. However, the most accurate method for calculating the necessary amount of correction required in sagittal imbalance surgery has not been identified. Consequently, complications and revision rates after these procedures are considerably high $[27,28]$, and the positive predictive value of sagittal balance correction is only approximately $75 \%$ [29]. The consequences of these failed interventions are frequently associated with adjacent segment degeneration and fractures as well as implant failures, followed by re-operations. Currently, surgical outcomes remain unsatisfactory for patients and surgeons, which raises the question whether the current methods of sagittal alignment evaluation are correct and the current surgical planning strategy adequate.

\section{Possible Reasons for the Failure of the Current Sagittal Deformity Correction Strategy}

\section{Radiological measurement errors}

Spinal alignment evaluated using a single lateral radiograph appears simplistic, and the accuracy of this method warrants critical reevaluation. Recent research has demonstrated that a slight rotation of the patient in the lateral projection significantly influences the value of spinal alignment parameters and changed arm positioning leads to a similarly strong bias [4,30-32]. Our investigations
[33] revealed that $51 \%$ of the 353 asymptomatic subjects displayed variations of $10 \%-20 \%$ in lumbar lordosis in six repeated standing phases and $29 \%$ showed variations of $>20 \%$. In sacrum orientation, $53 \%$ of the asymptomatic subjects displayed variations of $>20 \%$ and $31 \%$ showed variations of $>30 \%$. The reproducibility of repeated standing phases remained unaffected by age, sex, height, and body weight of patients. Patients with low back pain (LBP) displayed variability similar to that displayed by the asymptomatic cohort. The number of standing phases performed had no positive effect on the reproducibility. Therefore, the variability in standing is not predictable but is random and, thus, does not reflect any individual specific behavioral pattern that can be improved by methods such as repeated standing phases.

Given the high impact of a reproducible posture, several authors have provided practical recommendations for patient positioning during radiography. Dewi et al. [34] suggested a balancing plate that comprises a square board with a cylindrical disk at the center point attached at the center of the bottom. This design was aimed at realizing a forced balance in the sagittal and frontal planes. The balancing effect from the device forces the subject to stand in a balanced manner and directs the posture in a specific upright position. The authors demonstrated greater reproducibility when the balancing plate was used than that when standing on the ground by grasping a supporting bar. However, the position during standing on such an apparatus is not comparable to a naturally relaxed standing position. Moreover, Koreska et al. [35] implemented the "Throne" to reproduce the positioning of patients. However, this device only enabled the patient to be imaged in a sitting position, while the current standardized method for curvature measurement using radiography requires the patient to be in the standing position.

The patient's mental state also strongly affects the posture during radiologic imaging. An examiner can readily provoke a change in sagittal alignment by asking the patient to stand straighter. Depending on the routine method of the radiologist and the manner in which the patient is instructed, images can substantially vary even when the procedure is performed on the same individual. Spinal alignment is also influenced by the underlying disease. An example of this is a reversible positive sagittal imbalance in patients with symptomatic disc herniation [36]. Further, Suzuki et al. [37] suggested that positive sagittal balance decreases the epidural pressure through venous 
decompression, thereby causing patients to reflexively assume a more flexed posture. Therefore, functional- and posture-dependent back and leg pain appear important; however, the influence of these factors in evaluating sagittal alignment is currently underestimated.

Recent developments, including the EOS system (Biospace, Paris, France), have made it possible to obtain remarkable images to evaluate spinal deformities. EOS has been developed for orthopedic imaging and offers the benefit of the simultaneous measurement of posteroanterior and lateral images, allowing the three-dimensional (3D) reconstruction of the spine, pelvis, and lower limbs in an upright, weight-bearing (standing, seated, or squatting) position [38]. This system is indicated for pathologies that change under load, where the rotational deformity is relevant, or where radiation exposure is a concern because of the need for repeated radiographic imaging (the irradiation is $50 \%-80 \%$ less than that in conventional radiography [39]). However, currently, limited data are available about the clinical effectiveness of EOS. While the radiation dose is a concern for patients who require repeated imaging and appears to be a clear advantage offered by EOS over the standard radiographic technology, it is difficult to quantify the reductions in the radiation dose with this system in terms of patient health benefits [40]. While the accuracy of the 3D reconstruction obtained with EOS is equivalent to that obtained with twodimensional (2D) radiography images and computed tomography scans [41-43], the extraction of information is considerably more difficult from $3 \mathrm{D}$ images than from 2D images. Furthermore, manual measurement in 3D images requires navigation through a $3 \mathrm{D}$ image, which may be time consuming and difficult to interpret. However, the main limitations are that sagittal alignment parameters were developed for 2D images and transferred without modification to 3D images, which raises pertinent doubts regarding the usefulness of a new, expensive system, and the above-mentioned uncertainties for parameter acquisition from $2 \mathrm{D}$ images also apply to that from 3D images. Moreover, in the future, there is a need to develop automatic or robust methods of medical imaging that are cost effective and involve lower radiation exposure for evaluating spinal parameters. Finally, the above-mentioned problems related to a standardized and reproducible posture during radiography remain unresolved.

Several research groups currently use EOS; this is sometimes used in association with other imaging modalities or gait analysis for better diagnosis, treatment planning, and outcome. These studies are currently ongoing or are in the planning stage, and their findings may have important future implications.

We conclude that determining the necessary surgical correction using a single lateral radiograph is prone to biased measurements. Inter- and intra-individual differences and spine flexibility should be considered, and novel techniques for alignment evaluation should be developed.

\section{Non-spinal bias of sagittal alignment}

Sagittal spinal alignment does not depend only on the bony structures of the pelvis and the spine. When evaluating spinal alignment, changes in all parts and tissues of the musculoskeletal system, comprising the bone, muscle, cartilage, tendons, ligaments, and joints, must be considered. Therefore, the success of sagittal deformity correction also depends on non-spinal and non-pelvic confounding factors. Particularly, these considerations are crucial for patients with systemic disease. For example, corrective spinal surgeries performed in patients with neurodegenerative diseases, including Parkinson's disease, may fail after spinal alignment correction. The disease severity correlates with spinal deformity, and failure rates are considerably high [44]. Therefore, spinal alignment cannot be successfully corrected if the underlying disease is not simultaneously treated. Another example demonstrating the importance of a holistic approach to any spinal deformity is one with degenerative disease of the adjacent joints, including the hip [45].

\section{Evaluation and interpretation of compensatory effects}

The bilateral aspect of spinal alignment and the muscular/ musculoskeletal system becomes more apparent when considering the compensatory effects. It is well established that patients with positive sagittal plane deformities compensate not only through the spine (hyperlordosis of the cervical spine, reduction of thoracic kyphosis, retrolisthesis, and hyperextension of the lumbar spine) and pelvis (pelvic backtilt) but also through the adjacent joints, including the hip (extension), knee (flexion), and ankle (extension). The formulas for calculating the correction attempt to address these compensatory mechanisms by adding degrees to the correction. The vice versa alignment adaptations are relevant for any patient to compensate for 

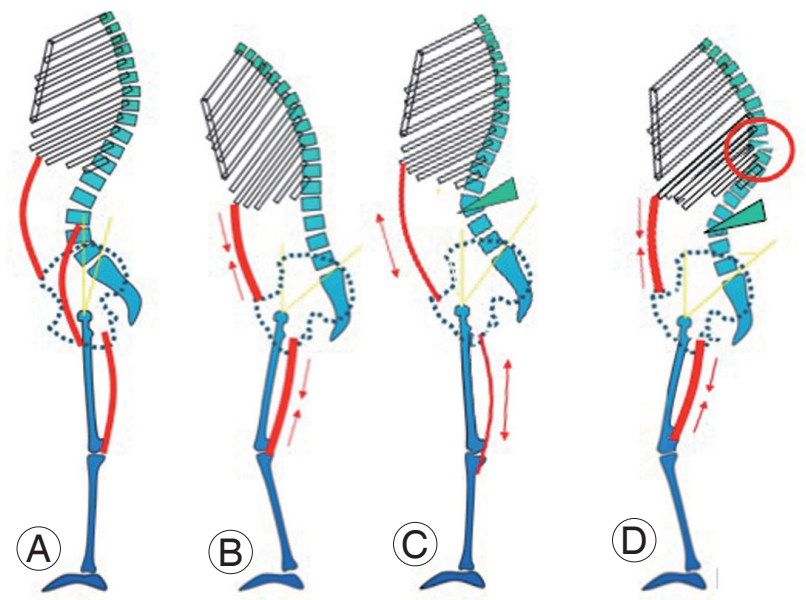

Fig. 2. The figure depicts $(\mathbf{A})$ the physiological and balanced spinal alignment in a young adult, $(\mathbf{B})$ the decompensated positive sagittal alignment of a patient with advanced age with a pelvic backtilt, a rigid thorax, and contractures of the hamstring and abdominal muscles, (C) the expected status after surgical correction and successful compensation (requires the patient's capability to achieve a pelvic fronttilt with an increase of the sacral slope and to secondary straighten the legs), and (D) proximal junctional failure following the inability to compensate as described. red arrows, musculature tension; green triangle, pedicle subtraction osteotomy; red circle, proximal fracture.

a spinal correction procedure. However, to our knowledge, thus far, the patient's ability to compensate for the correction postoperatively has not been considered in any study. In addition, neither clinical nor radiological evaluation methods of such compensatory capabilities have been established. Furthermore, pelvic compensation is limited not only by its anatomical composition, including the bony range of motion in the hip joints, but also by contract musculature. In cases of severely shortened spinofemoral muscles (hamstring contractures), complete compensation cannot be achieved. Therefore, compensatory capabilities significantly vary between individuals and decrease, particularly with aging, as illustrated in Fig. 2.

We may consider a different perspective wherein the soft tissue changes are the actual underlying cause of the development of sagittal misalignment over time. In this case, spinal misalignment would represent a bony adaptation to the soft tissue changes. By the correction of the bony skeleton, a new stimulus toward adaptation and, therefore, decompensation would be generated. Few current biomechanical models have considered this.

Mental status and the associated factors more frequently influence the ability to compensate for surgical spinal alignment correction than soft or hard tissues. Patients have an intrinsic perspective regarding their individual postures, and they become accustomed to this over time. Similar to much younger patients with adolescent scoliosis undergoing corrective surgery, they need to postoperatively learn about an upright and straight posture. If they are unable to learn this, they tend to revert to their previous gravity line, causing sagittal imbalance, adjacent level fractures, or implant failure.

In summary, there is a need to develop clinical and/ or biomechanical tools for better evaluation and for the further discrimination of soft and hard tissue compensatory abilities of the spine and its environment. Moreover, the existing biomechanical models need to be adapted to these compensatory abilities, for example, muscle contractures.

\section{Future Research in the Assessment of Spinal Alignment}

Although considerable advances have been made in the assessment of spinal alignment, limited clinical outcomes have suggested that further investigations are urgently needed. The following objectives should be an integral part of future research: (1) physiological aging process of the spine with regard to spinal alignment and body balance; (2) development of biomechanical assessment tools for functional spinal alignment; and (3) development of biomechanical models and tools for predicting individual compensatory possibilities and adapted correction planning.

Before the analyses of deformity correction, research efforts should be directed toward the physiological aging process of the spine and the matching of individual spinal alignment and body balance. Current knowledge regarding age-related losses in lumbar spinal lordosis and positive sagittal balance in adults is limited $[4,7,46]$.

However, a physiologically positive balance in adults could change the threshold for, and the extent of, correction and explain the current high failure rate. This change might be attributable not only to the spine but also to the musculoskeletal system, as in contractures of the ischiocrural muscles or arthritis of the adjacent joints $[47,48]$. This raises the question, "Are we overcorrecting patients?" Blondel et al. [49] demonstrated that postoperative patient alignment distribution was almost equal between successful restoration (neutral position), remaining positive (malalignment), and overcorrection. Moreover, the need for 
information about the natural course of spinal alignment during aging becomes more apparent because acute proximal junctional failures correlate with preoperative positive sagittal balance [50]. In keeping with these findings, it has been demonstrated that the revision rate progressively increases as per the follow-up time [51]. Future research should be directed toward defining a sagittal profile according to the patient's age. Therefore, we propose to differentiate between sagittal alignment and spinal or body balance. Spinal alignment is assessed using standardized measurements and parameters that are important for inter-individual comparisons. The biomechanical evaluation of a patient's balance over a defined time and exercise may provide insights into an individual's balance profile. Individually matching both spinal alignment and balance may decrease the failure rates and lead to surgical success.

Current assessment method of spinal alignment using a single static radiograph is not adequately accurate when considering spinal flexibility and the overall static and functional demands on the spine. Surgical treatment should be adapted as per these demands. Therefore, to plan a successful surgery, clinicians will require a more extensive analysis of the patient's functional spinal alignment. However, because numerous radiographies would be required, this would increase the radiation level to ethically unacceptable levels. Therefore, novel non-radiological techniques that can evaluate the spinal profile during regular daily life should be implemented in routine clinical practice. In a recent study on 208 asymptomatic subjects, we determined the shape of the lumbar spine over a period of 24 hours using a non-invasive measurement tool [52]. The mean lumbar lordosis was subsequently compared with the lordosis achieved during standing for short term, similar to radiological assessment. We found a difference of $23^{\circ}$ in lumbar lordosis between short-term examinations and average "real-life" evaluations during the day, which clearly indicates that lumbar lordosis is highly dynamic during the day, and on an average, considerably lower over the entire day than that in the standing position alone. This basic knowledge is important for surgical planning and potential improvement of the understanding of outcomes of different interventions and postoperative problems.

Previously, sagittal spinal alignment was mainly studied in the standing position in association with factors such as aging and degenerative lumbar disease. However, with the development of modern computer technology, the sitting position has currently become the most common posture in workplace. Office workers reportedly spend $80 \%$ of their work time in the sitting position. Therefore, knowledge about the normal sitting sagittal spinal alignment is important for the long-term effects of the sitting position and for the prevention of LBP in daily life. Endo et al. [22] showed that lumbar lordosis decreased by approximately $50 \%$ and pelvic tilt increased by approximately $25 \%$ in the sitting position compared to those in the standing position.

Certain standardized movements should be measured to enable inter-individual comparisons. The human skeleton is a factor that influences body posture and spinal alignment. Measurements should evaluate not only structural differences related to the bones but also those related to soft tissues, particularly the muscles. Furthermore, recent studies have demonstrated the need to explore the relationship between reduced physical performance and the sensory and cognitive perceptions of pain. The results of these studies strongly support the hypothesis that spinal physical capacity in chronicity is not solely explained by the sensory perception of pain. The anticipation of pain and the fear avoidance belief about physical activities were the strongest predictors of variation in physical performance. Therefore, they should be integrated into the clinical assessment of chronic LBP.

However, conservative treatment fails in several patients with spine-related deformities, indicating surgical intervention. In such cases, in addition to planning the extent, type, and localization of corrections, the individual compensatory capabilities for the correction must also be preoperatively evaluated. Although Lazennec et al. [53] made initial attempts to measure pelvic compensatory effects in patients, to our knowledge, no standardized method has been established to estimate the overall individual correction potential. The development of biomechanical measurement tools and technologies for the evaluation and prediction of individual compensatory capabilities of the spine, pelvis, and legs is crucial. There is a question regarding whether all musculoskeletal compensatory capabilities can be summarized. This must equal or surpass the planned deformity correction. Thus, we suggest that the correction should be inversely proportional to the degree of rigidity of the deformity and the musculoskeletal environment.

In the future, combined clinical, radiological, psychological, and biomechanical analyses are warranted to 
thoroughly plan orthopedic spine deformity surgeries. Detailed insights into spinal alignment and individual spinal or body balance will facilitate clinicians to overcome the current limitations and improve routine patient care.

\section{Conclusions}

In past decades sagittal balance has been extensively studied and a high relevance in the surgical decision making process has been pointed out. However, critical evaluation of the ongoing research is warranted and established treatment strategies should be refined. Further research directions should identify current shortcomings regarding the compensatory potential of patients. In future, these could reduce specifically mechanical complications following deformity surgery. Furthermore the current spinal knowledge of alignment and balance should be extrapolated to the entire musculoskeletal system. The soft tissue, especially muscles and ligaments, should attract more attention.

\section{Conflict of Interest}

No potential conflict of interest relevant to this article was reported.

\section{Acknowledgments}

This study was financially supported by the Bundesinstitut für Sportwissenschaften, BiSp (MiSpEx - Network).

\section{References}

1. Schunke M, Schulte E, Schumacher U. Prometheus Allgemeine Anatomie und Bewegungssystem: LernAtlas der Anatomie. 3. Auflage. Stuttgart: Gerorg Thieme Verlag; 2011.

2. Stagnara P, De Mauroy JC, Dran G, et al. Reciprocal angulation of vertebral bodies in a sagittal plane: approach to references for the evaluation of kyphosis and lordosis. Spine (Phila Pa 1976) 1982;7:335-42.

3. Vaz G, Roussouly P, Berthonnaud E, Dimnet J. Sagittal morphology and equilibrium of pelvis and spine. Eur Spine J 2002;11:80-7.

4. Vialle R, Levassor N, Rillardon L, Templier A, Skalli W, Guigui P. Radiographic analysis of the sagittal alignment and balance of the spine in asymptomatic subjects. J Bone Joint Surg Am 2005;87:260-7.

5. Roussouly P, Gollogly S, Berthonnaud E, Dimnet J. Classification of the normal variation in the sagittal alignment of the human lumbar spine and pelvis in the standing position. Spine (Phila Pa 1976) 2005;30:346-53.

6. Faundez A, Roussouly P, Le Huec JC. Sagittal balance of the spine: a therapeutic revolution. Rev Med Suisse 2011;7:2470-4.

7. Hammerberg EM, Wood KB. Sagittal profile of the elderly. J Spinal Disord Tech 2003;16:44-50.

8. Amonoo-Kuofi HS. Changes in the lumbosacral angle, sacral inclination and the curvature of the lumbar spine during aging. Acta Anat (Basel) 1992;145:3737.

9. Tuzun C, Yorulmaz I, Cindas A, Vatan S. Low back pain and posture. Clin Rheumatol 1999;18:308-12.

10. Lin RM, Jou IM, Yu CY. Lumbar lordosis: normal adults. J Formos Med Assoc 1992;91:329-33.

11. Schwab F, Lafage V, Boyce R, Skalli W, Farcy JP. Gravity line analysis in adult volunteers: age-related correlation with spinal parameters, pelvic parameters, and foot position. Spine (Phila Pa 1976) 2006;31:E959-67.

12. Hansson T, Bigos S, Beecher P, Wortley M. The lumbar lordosis in acute and chronic low-back pain. Spine (Phila Pa 1976) 1985;10:154-5.

13. Gelb DE, Lenke LG, Bridwell KH, Blanke K, McEnery KW. An analysis of sagittal spinal alignment in 100 asymptomatic middle and older aged volunteers. Spine (Phila Pa 1976) 1995;20:1351-8.

14. Jackson RP, McManus AC. Radiographic analysis of sagittal plane alignment and balance in standing volunteers and patients with low back pain matched for age, sex, and size: a prospective controlled clinical study. Spine (Phila Pa 1976) 1994;19:1611-8.

15. La Grone MO. Loss of lumbar lordosis: a complication of spinal fusion for scoliosis. Orthop Clin North Am 1988;19:383-93.

16. Pellet N, Aunoble S, Meyrat R, Rigal J, Le Huec JC. Sagittal balance parameters influence indications for lumbar disc arthroplasty or ALIF. Eur Spine J 2011;20 Suppl 5:647-62.

17. Troke M, Moore AP, Maillardet FJ, Cheek E. A normative database of lumbar spine ranges of motion. Man Ther 2005;10:198-206.

18. Van Herp G, Rowe P, Salter P, Paul JP. Three-dimensional lumbar spinal kinematics: a study of range of 
movement in 100 healthy subjects aged 20 to $60+$ years. Rheumatology (Oxford) 2000;39:1337-40.

19. Peleg S, Dar G, Medlej B, et al. Orientation of the human sacrum: anthropological perspectives and methodological approaches. Am J Phys Anthropol 2007;133:967-77.

20. Peleg S, Dar G, Steinberg N, Peled N, Hershkovitz I, Masharawi Y. Sacral orientation revisited. Spine (Phila Pa 1976) 2007;32:E397-404.

21. Boulay C, Tardieu C, Hecquet J, et al. Sagittal alignment of spine and pelvis regulated by pelvic incidence: standard values and prediction of lordosis. Eur Spine J 2006;15:415-22.

22. Endo K, Suzuki H, Nishimura H, Tanaka H, Shishido T, Yamamoto K. Sagittal lumbar and pelvic alignment in the standing and sitting positions. J Orthop Sci 2012;17:682-6.

23. Roussouly P, Nnadi C. Sagittal plane deformity: an overview of interpretation and management. Eur Spine J 2010;19:1824-36.

24. Kim H, Kim HS, Moon ES, et al. Scoliosis imaging: what radiologists should know. Radiographics 2010;30:1823-42.

25. Glassman SD, Berven S, Bridwell K, Horton W, Dimar JR. Correlation of radiographic parameters and clinical symptoms in adult scoliosis. Spine (Phila Pa 1976) 2005;30:682-8.

26. Berjano P, Cecchinato R, Damilano M, Morselli C, Sansone V, Lamartina C. Preoperative calculation of the necessary correction in sagittal imbalance surgery: validation of three predictive methods. Eur Spine J 2013;22 Suppl 6:S847-52.

27. Pichelmann MA, Lenke LG, Bridwell KH, Good CR, O'Leary PT, Sides BA. Revision rates following primary adult spinal deformity surgery: six hundred forty-three consecutive patients followed-up to twenty-two years postoperative. Spine (Phila Pa 1976) 2010;35:219-26.

28. Smith JS, Sansur CA, Donaldson WF 3rd, et al. Short-term morbidity and mortality associated with correction of thoracolumbar fixed sagittal plane deformity: a report from the Scoliosis Research Society Morbidity and Mortality Committee. Spine (Phila Pa 1976) 2011;36:958-64.

29. Lafage V, Bharucha NJ, Schwab F, et al. Multicenter validation of a formula predicting postoperative spinopelvic alignment. J Neurosurg Spine 2012;16:15-
21.

30. Aota Y, Saito T, Uesugi M, Kato S, Kuniya H, Koh R. Optimal arm position for evaluation of spinal sagittal balance. J Spinal Disord Tech 2011;24:105-9.

31. Hayashi K, Upasani VV, Pawelek JB, et al. Threedimensional analysis of thoracic apical sagittal alignment in adolescent idiopathic scoliosis. Spine (Phila Pa 1976) 2009;34:792-7.

32. Schmidt J, Gassel F. Clinical use of the simple 3Dcalculation in scoliosis. Skeletal Radiol 1994;23:43-8.

33. Schmidt H, Bashkuev M, Weerts J, et al. How do we stand?: variations during repeated standing phases of asymptomatic subjects and low back pain patients. J Biomech 2018;70:67-76.

34. Dewi DE, Veldhuizen AG, Burgerhof JG, et al. Reproducibility of standing posture for X-ray radiography: a feasibility study of the BalancAid with healthy young subjects. Ann Biomed Eng 2010;38:3237-45.

35. Koreska J, Schwentker EP, Albisser AM, Gibson DA, Mills RH. A simple approach to standardized spinal radiographs. Med Instrum 1978;12:59-63.

36. Fujii K, Kawamura N, Ikegami M, Niitsuma G, Kunogi J. Radiological improvements in global sagittal alignment after lumbar decompression without fusion. Spine (Phila Pa 1976) 2015;40:703-9.

37. Suzuki H, Endo K, Kobayashi H, Tanaka H, Yamamoto $\mathrm{K}$. Total sagittal spinal alignment in patients with lumbar canal stenosis accompanied by intermittent claudication. Spine (Phila Pa 1976) 2010;35:E344-6.

38. Morvan G, Mathieu P, Vuillemin V, et al. Standardized way for imaging of the sagittal spinal balance. Eur Spine J 2011;20 Suppl 5:602-8.

39. Melhem E, Assi A, El Rachkidi R, Ghanem I. EOS((R)) biplanar X-ray imaging: concept, developments, benefits, and limitations. J Child Orthop 2016;10:1-14

40. Wade R, Yang H, McKenna C, Faria R, Gummerson $\mathrm{N}$, Woolacott N. A systematic review of the clinical effectiveness of EOS 2D/3D X-ray imaging system. Eur Spine J 2013;22:296-304.

41. Dubousset J, Charpak G, Dorion I, et al. A new 2D and $3 \mathrm{D}$ imaging approach to musculoskeletal physiology and pathology with low-dose radiation and the standing position: the EOS system. Bull Acad Natl Med 2005;189:287-97.

42. Somoskeoy S, Tunyogi-Csapo M, Bogyo C, Illes T. Accuracy and reliability of coronal and sagittal spi- 
nal curvature data based on patient-specific threedimensional models created by the EOS $2 \mathrm{D} / 3 \mathrm{D}$ imaging system. Spine J 2012;12:1052-9.

43. Al-Aubaidi Z, Lebel D, Oudjhane K, Zeller R. Threedimensional imaging of the spine using the EOS system: is it reliable?: a comparative study using computed tomography imaging. J Pediatr Orthop B 2013;22:409-12.

44. Oh JK, Smith JS, Shaffrey CI, et al. Sagittal spinopelvic malalignment in Parkinson disease: prevalence and associations with disease severity. Spine (Phila Pa 1976) 2014;39:E833-41.

45. Parratte S, Pagnano MW, Coleman-Wood K, Kaufman KR, Berry DJ. The 2008 Frank Stinchfield award: variation in postoperative pelvic tilt may confound the accuracy of hip navigation systems. Clin Orthop Relat Res 2009;467:43-9.

46. Dreischarf M, Albiol L, Rohlmann A, et al. Agerelated loss of lumbar spinal lordosis and mobility: a study of 323 asymptomatic volunteers. PLoS One 2014;9:e116186.

47. Lee CS, Park SJ, Chung SS, Lee KH. The effect of simulated knee flexion on sagittal spinal alignment: novel interpretation of spinopelvic alignment. Eur Spine J 2013;22:1059-65.
48. Penning L. Psoas muscle and lumbar spine stability: a concept uniting existing controversies: critical review and hypothesis. Eur Spine J 2000;9:577-85.

49. Blondel B, Schwab F, Bess S, et al. Posterior global malalignment after osteotomy for sagittal plane deformity: it happens and here is why. Spine (Phila $\mathrm{Pa}$ 1976) 2013;38:E394-401.

50. Smith MW, Annis P, Lawrence BD, Daubs MD, Brodke DS. Acute proximal junctional failure in patients with preoperative sagittal imbalance. Spine J 2015;15:2142-8.

51. Maier SP, Smith JS, Schwab FJ, et al. Revision surgery after 3-column osteotomy in 335 patients with adult spinal deformity: intercenter variability and risk factors. Spine (Phila Pa 1976) 2014;39:881-5.

52. Dreischarf M, Pries E, Bashkuev M, Putzier M, Schmidt H. Differences between clinical "snap-shot" and "real-life" assessments of lumbar spine alignment and motion: what is the "real" lumbar lordosis of a human being? J Biomech 2016;49:638-44.

53. Lazennec JY, Brusson A, Folinais D, Zhang A, Pour AE, Rousseau MA. Measuring extension of the lumbar-pelvic-femoral complex with the EOS system. Eur J Orthop Surg Traumatol 2015;25:1061-8. 